\title{
Influência da Energia de Soldagem do Processo RSW sobre as Propriedades Magnéticas e Tensões Residuais no Aço AISI 444
}

\author{
Adriano Cássio Baldim ${ }^{1}$, Sebastião Carlos da Costa $^{1}$, Manoel Ribeiro da Silva ${ }^{1}$, Claudiney de Sales Pereira Mendonça ${ }^{1}$, \\ Marcos Cirilo dos Santos ${ }^{1}$, Gilbert Silva ${ }^{1}$, Vander Alkmin dos Santos Ribeiro ${ }^{1}$ \\ ${ }^{1}$ Universidade Federal de Itajubá - UNIFEI, Instituto de Engenharia Mecânica/Laboratório de Soldagem, Itajubá, MG, Brasil.
}

Recebido: 22 Out., 2017

Aceito: 14 Mar., 2018

E-mails: adriano_baldim@yahoo. com.br (ACB), sccosta@unifei.edu.br (SCC), mrsilva@unifei.edu.br (MRS), claudiney.sales@unifei.edu.br (CSPM), marcos.cirilo@unifei.edu.br (MCS), gilbert@unifei.edu.br (GS), vanderalkmin@gmail.com (VASR)
Resumo: Os aços inoxidáveis ferríticos vêm ganhando cada vez mais espaço na indústria, devido principalmente a sua grande durabilidade e aplicabilidade. Nos últimos anos, o crescente avanço tecnológico da indústria metal mecânica, possibilitou o desenvolvimento de novas ligas e processos de soldagem deste metal. Em meio a estes processos, a soldagem por resistência tem sido muito utilizada por possibilitar rapidez com custo baixo. Muito embora existam poucas informações disponíveis na literatura, é reconhecido que a união pela mescla da atuação simultânea de calor e deformação, presentes no processo de soldagem por resistência, podem interferir diretamente nas propriedades magnéticas e mecânicas dos aços inoxidáveis ferríticos. Assim, uma avaliação destas características em produtos manufaturados torna-se importante em determinados setores industriais, notadamente nos de equipamentos eletrônicos e metrológicos. O objetivo desse artigo é a observação das tensões residuais e propriedades magnéticas em diferentes níveis de energia de soldagem e deformação no processo de soldagem a ponto por resistência elétrica em chapas de aço AISI 444. Os resultados mostraram que a energia de soldagem e deformação afetam as propriedades magnéticas, por outro lado não existe influência substancial na tensão residual.

Palavras-chave: Aço inoxidável ferrítico; Energia de soldagem; Tensão residual; Propriedades magnéticas; Soldagem a ponto por resistência.

\section{The Influence of the Welding Energy on the Residual Stress and Magnetic Properties of Steel AISI 444 Welded by the RSW Process}

\begin{abstract}
Ferritic stainless steel has been gaining space in the industry, due mainly to its durability and applicability. In recent years, the growing technological advances of the metalworking industry, enabled the development of new alloys and welding processes of this metal. In the midst of these processes, resistance spot welding has been largely used due to the very short process time with low cost. Although there is a lack of available information in the literature, the union of materials by the mixture of the simultaneous action of heat and deformation, present in the resistance spot welding, can affects the mechanical and magnetic properties directly of ferritic stainless steels. Thus, an evaluation of these characteristics in manufactured products becomes important in certain industrial sectors, especially in electronic and metrological equipment. The main objective of this article is the observation of residual stresses and magnetic properties at different levels of welding energy and deformation in the resistance spot welding process steel AISI 444. The results showed that welding and deformation energy affect magnetic properties, on the other hand there is no substantial influence on the residual stress.
\end{abstract}

Key-words: Ferritic stainless steel; Welding energy; Residual stresses; Magnetic properties; Resistance spot welding.

\section{Introdução}

As investigações científicas e tecnológicas ligadas aos processos de soldagem são interessantes para o avanço tecnológico na área de fabricação mecânica. Por se tratar de um setor industrial de produção, grandes investimentos são necessários e a redução de custos assume um papel importante. Neste sentido, dentre os processos de soldagem existentes, o processo de soldagem a ponto por resistência elétrica também denominado RSW (do inglês Resistance Spot Welding) se destaca como um dos mais utilizados nas indústrias metal mecânica [1]. Isto ocorre devido a sua simplicidade, robustez e baixo custo de aplicação, comparativamente a outros processos de soldagem a arco elétrico
Este é um artigo publicado em acesso aberto (Open Access) sob a licença Creative Commons Attribution Non-Commercial, que permite uso, distribuição e reprodução em qualquer meio, sem restriçōes desde que sem fins comerciais e que 0 trabalho original seja corretamente citado. 
como MIG/MAG, TIG entre outros [2]. Dentre os diversos materiais existentes no mercado, os aços inoxidáveis vêm ganhando cada vez mais espaço dentro de setores específicos da indústria, notadamente nos de equipamentos eletrônicos e metrológicos. Neste cenário, estão aços inoxidáveis ferríticos, especialmente por possuir baixo teor de níquel proporcionam um menor custo. Aliada a uma boa soldabilidade quando estabilizados por $\mathrm{Ti}$ e $\mathrm{Nb}$, induz a uma atratividade por estes materiais [3-5].

Considerando a natureza do material bem como parâmetros no curso de seu processamento, o comportamento magnético dos aços inoxidáveis ferríticos pode ser influenciado por diversos fatores. Sabe-se para tanto que, materiais com propriedades magnéticas submetidos à deformação e calor, os campos magnéticos sofrem alterações significativas. Contudo, tendo em vista que o princípio básico do processo de soldagem a ponto por resistência elétrica se concentra no efeito Joule, e por consequência a união de materiais ocorra pela mescla da atuação simultânea de energia de soldagem e pressão, pode se pressupor que as propriedades magnéticas possam ser alteradas [6]. Todavia, alguns autores como Vashista \& Paul [7] perceberam que as tensões residuais, a dureza e a microestrutura influenciam no domínio magnético dos materiais ferríticos. Neste sentido, Tavares et al. [8] avaliaram que a formação de precipitados aufere pequeno aumento na dureza magnética. Dentre as propriedades magnéticas destes materiais, os pesquisadores notaram que a magnetização de saturação e a permeabilidade magnética aumentam com as tensões residuais. Para tanto, uma avaliação das propriedades magnéticas do material e das tensões residuais após a soldagem, podem revelar correlação com a deformação e a energia de soldagem aplicada. Nas aplicações de precisão como implantes biomédicos [9], as características eletromagnéticas ganham destaque, principalmente quando há interferência em aparelhos que mantêm a vida humana. Como exemplo, a inibição do funcionamento de um cardioversor-desfribilador implantável [10] com a elevação do campo magnético coercivo. Já no setor automotivo, a natureza magnética mole dos aços ferríticos, definida pela facilidade de magnetização e desmagnetização, melhora o tempo de resposta das válvulas de injeção de combustível. Atualmente esta propriedade tem sido um grande desafio da indústria automobilística a fim de aumentar a eficiência dos motores durante a combustão [11]. Com base nestes aspectos, faz-se necessário uma atenção especial ao procedimento de soldagem com intuito de evitar alterações nas propriedades magnéticas e também, nas tensões residuais presentes nos aços inoxidáveis ferríticos [12,13]. Neste contexto, este artigo tem por objetivo a observação da influência da energia de soldagem sobre as propriedades magnéticas e tensões residuais considerando a parametrização do equipamento e deformação no processo de soldagem a ponto por resistência elétrica em chapas de aço AISI 444. Como forma de avaliação da influência dos parâmetros, as deformações referentes à atuação do eletrodo de cobre foram avaliadas através de um sensor de deslocamento. A energia de soldagem gerada pelo efeito Joule durante o processo RSW foi calculada por meio de equações. As tensões residuais foram determinadas por meio da técnica de difratometria de raios-X. Finalmente as propriedades magnéticas das amostras soldadas foram avaliadas através de um magnetômetro de amostra vibrante.

\section{Materiais e Métodos}

Nos testes de soldagem através do processo RSW, foram utilizados eletrodos de Cobre Cromo Zircônio, de cone truncado $15^{\circ}$, diâmetro da ponta de $5 \mathrm{~mm}$. A espessura da chapa utilizada para o experimento foi de $1 \mathrm{~mm}$. Para a preparação das amostras, foram utilizadas duas chapas de aço inoxidável ferrítico AISI 444, cuja composição química é mostrada na Tabela 1.

As soldagens foram realizadas em uma máquina de solda a ponto estacionária marca Presol Transweld, modelo TWPRV50 com potência nominal de 5 kVA e corrente máxima de 6 kA, com acionamento pneumático. Acoplado ao equipamento de soldagem a ponto, foi utilizado um transdutor diferencial de deslocamento linear (LVDT) com o intuito de medir a deformação causada pela ação dos eletrodos no material soldado. Para a execução das soldagens, o equipamento foi ajustado com níveis de pressão $P$ alternando entre 0,4 MPa e 0,6 Mpa. Sendo a definição de pressão: força por unidade de área [2], torna-se possível calcular o valor da força aplicada entre os eletrodos ao considerar o diâmetro do embolo do pistão de acionamento pneumático do equipamento igual a $8 \mathrm{~cm}$. Os níveis de corrente I ajustados foram $4200 \mathrm{~A}$ e $5400 \mathrm{~A}$ e os tempos de soldagem t ajustados foram 10 e 30 ciclos de soldagem, conforme mostrado na Tabela 2. Para uma frequência de $60 \mathrm{~Hz}$ de corrente elétrica de soldagem, 60 ciclos de parametrização do equipamento equivalem ao tempo de $1 \mathrm{~s}$.

Tabela 1. Composição química do aço inoxidável AISI 444. Fonte: [8].

\begin{tabular}{cccccccccc}
\hline Material (\%) & $\mathbf{C}$ & $\mathbf{C r}$ & $\mathbf{N i}$ & $\mathbf{M o}$ & $\mathbf{T i}$ & $\mathbf{N b}$ & $\mathbf{S i}$ & $\mathbf{N}$ & $\mathbf{F e}$ \\
Aço AISI 444 & 0,015 & 17,56 & 0,20 & 1,86 & 0,13 & 0,18 & 0,54 & 0,0123 & Balanceado \\
\hline
\end{tabular}


Influência da Energia de Soldagem do Processo RSW sobre as Propriedades Magnéticas e Tensões Residuais no Aço AISI 444

Tabela 2. Parâmetros de soldagem RSW utilizado.

\begin{tabular}{cccc}
\hline Amostras & t & I & $\begin{array}{c}\text { P1(*) (Força } \\
\text { aplicada [N]) }\end{array}$ \\
\hline 0 & S & A & MPa \\
1 & - & - & - \\
2 & 0,166 & 4200 & $0,4(2000)$ \\
3 & 0,500 & 4200 & $0,4(2000)$ \\
4 & 0,166 & 5400 & $0,4(2000)$ \\
5 & 0,166 & 4200 & $0,6(3015)$ \\
6 & 0,500 & 4200 & $0,6(3015)$ \\
7 & 0,166 & 5400 & $0,6(3015)$ \\
\hline
\end{tabular}

*O valor P1 se trata do número de regulagem da pressão no equipamento, mas o valor da força entre os eletrodos correspondente à regulagem, é apresentado entre parênteses.

Inicialmente as 8 amostras foram seccionadas em dimensões de $6 \times 6 \mathrm{~mm}^{2}$ conforme mostrado na Figura 1a. Esta dimensão foi propositalmente escolhida com intuito de obter maior deformação e energia de soldagem culminando em maior variação das propriedades magnéticas. Em seguida procedeu-se a soldagem a ponto somente de 7 amostras conforme detalhamento mostrado na Figura 1b. A amostra 0 foi deixada no seu estado natural como referência inicial de propriedades magnéticas e de tensão residual. Após avaliação da tensão residual, as amostras foram arredondadas por meio de um processo mecânico até atingir o formato ilustrado na Figura 1c, adequado para avaliação das propriedades magnéticas [14].

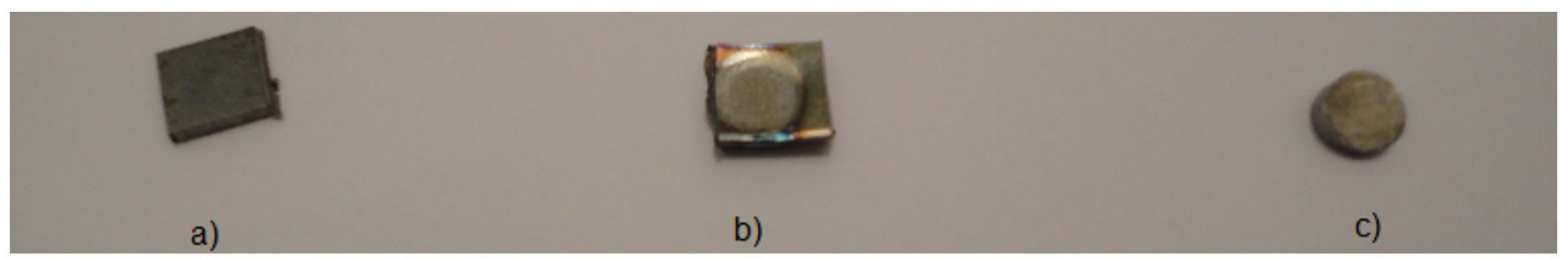

Figura 1. a) Amostra cortada em $6 \times 6 \mathrm{~mm}^{2}$, b) amostras sobrepostas e soldadas, c) amostra preparada para a medição magnética.

Em um condutor elétrico a quantidade de calor gerado depende de três fatores: corrente elétrica, resistência do condutor e duração da corrente. Na soldagem RSW a geração de calor, ocorre pelo efeito Joule devido à resistência do conjunto à passagem de corrente ocorrendo em maior quantidade no ponto de maior resistência, localizado entre as superfícies das chapas a serem soldadas. O tempo de soldagem deve ser suficiente para que o material atinja o ponto de fusão formando o ponto de soldagem [1,2]. Sabe-se para tanto que, o calor gerado no efeito Joule pode ser calculado pela Equação da energia de soldagem [2] conforme Equação 1.

$$
Q=I^{2} R t
$$

Na Equação 1, Q representa a energia térmica desenvolvida no ato da solda [Joule]; t o tempo de duração da passagem da corrente elétrica [s]; $R$ a resistência do conjunto a passagem de corrente [ohms] e I o valor da intensidade da corrente elétrica que passa através do metal [A]. A resistência no processo RSW é um parâmetro complexo de se determinar. Desta forma, considerando que a maior resistência ocorre entre as chapas [1], será utilizado o método de determinação da resistência dinâmica descrito por Tan et al. [15] como uma idealização teórica da situação física real que ocorre em R. Este método tem seu foco nas resistências das chapas e no ponto de maior geração de energia. Assim, a variável R da Equação 1 pode ser discriminada pela Equação 2.

$$
R=R_{F}+R_{C}+R_{B}
$$


Na Equação $2, R_{F}$ representa a resistência oferecida pela contaminação na superfície das chapas ou filmes de revestimento. Ao utilizar as chapas isentas de contaminantes e revestimento, a resistência $R_{F}$ pode ser considerada igual a 0 [15]. $R_{C}$ representa a resistência entre as superfícies do metal soldado e varia em função pressão e/ou força dos eletrodos aplicada durante a soldagem, conforme descrito na Equação 3.

$$
R_{C}=0,89 \rho\left(\frac{\varepsilon H}{n F}\right)^{\frac{1}{2}}
$$

Onde $\rho$ representa a resistividade elétrica do material $(0,0000620 \mathrm{Ohm} . \mathrm{cm})[16], \varepsilon$ o fator de pressão que pode variar entre 0,2 a 1,0 na superfície de contato entre chapas. Neste exemplo, o valor de $\varepsilon=0,2$ é indicado para superfícies elásticas, já o valor de $\varepsilon=1,0$ é indicado para superfícies completamente plásticas ou dúcteis. Para este estudo foi utilizado o valor de $\varepsilon=0,7$ como uma aproximação razoável para o aço inoxidável utilizado. Em seguida, representada por $\mathrm{H}$ está a dureza (95 HRB) [16] do material a ser soldado. Posteriormente, denotado por $n$ (igual a 1) está o número de pontos de soldagem que cada amostra receberá. Por último, representado por $\mathrm{F}[\mathrm{N}]$ está a força do eletrodo. Totalizando o detalhamento da Equação 2 tem-se $R_{B}$ que representa a resistência elétrica da chapa de metal, determinada por meio da Equação 4. Neste cenário, $\rho$ representa a resistividade elétrica do material [Ohm-cm]; $l$ representa a soma das espessuras das chapas (caminho percorrido pela corrente elétrica) [cm], A é a área da ponta do eletrodo $\left[\mathrm{cm}^{2}\right]$ por onde a corrente de soldagem é aplicada na chapa de metal $[2,15]$.

$$
R_{B}=\rho \frac{l}{A}
$$

As propriedades de tensão residual foram analisadas por meio do equipamento de difratometria de raios $X$, modelo X'PERT PRO da PANalytical. Os dados foram coletados utilizando uma geometria de feixe paralelo, foco pontual, com uma voltagem de $40 \mathrm{kV}$ e corrente de $45 \mathrm{~mA}$. Após as análises de tensões residuais as amostras foram arredondadas até adquirirem o formato de pequenos discos de aproximadamente $5 \mathrm{~mm}$ de diâmetro (Figura 1c). Este procedimento é necessário para a correção do campo desmagnetizante [14]. Para obtenção da curva de magnetização versus campo aplicado, os experimentos foram realizados em um magnetômetro de amostra vibrante VSM, marca Lake Shore, com um campo máximo aplicado de $\pm 14 \mathrm{kG}$. Em seguida foram pesadas em uma balança com precisão de $0,00001 \mathrm{~g}$ com intuito de obter os resultados de magnetização em função do peso. A microestrutura foi analisada por meio de um microscópio ótico e um microscópio eletrônico de varredura com o propósito observar o crescimento dos grãos e a formação de precipitados. $O$ ataque ácido com o reagente Villela foi realizado por $90 \mathrm{~s}$ conforme descrito por Tavares [7]. Outros reagentes como behara modificado por $10 \mathrm{~s}$ e ácido oxálico eletrolítico por $40 \mathrm{~s}$ com $10 \mathrm{~V}$ de tensão, também foram averiguados. Por último, uma análise das correlações [17] avaliadas pela variação entre energia de soldagem em relação à variação das propriedades magnéticas e de tensão residual foram processadas por meio do software Minitab ${ }^{\circledR}$.

\section{Resultados e Discussão}

Após realização das soldagens conforme condições estabelecidas na Tabela 2, as amostras foram submetidas à avaliação das propriedades magnéticas. Na Tabela 3, representado por L estão os resultados obtidos para a deformação causada pela ação dos eletrodos no material soldado, conjuntamente com as propriedades magnéticas do aço AISI 444 constituídas pelo campo coercivo Hc, pela magnetização remanente $\mathrm{Mr}$, pela magnetização de saturação Ms, pela permeabilidade magnética $\mu$ e pela área da histerese A. Por último estão o nível de tensão residual $\sigma$ e finalmente a energia de soldagem $Q$ calculada por meio das Equações 1 e 2 sendo esta última fragmentada pelas Equações de resistividade 3 e 4, já considerando os valores de $\mathrm{F}, \mathrm{H}$ e $n$. Os valores negativos de $\sigma$ e de $A$, representam a tensão residual compressiva e as perdas de magnetização pela área do laço da histerese [7]; $\mathrm{t}$, l e $\mathrm{F}$ seguem conforme estabelecidos anteriormente na Tabela 2.

Observa-se na Tabela 3 que, para um mesmo nível de l e t verifica-se um aumento de Q para níveis menores de $F$. Este fato reforça a influência de $F$ (força entre os eletrodos) na resistência à passagem de corrente entre chapas $[1,2,15]$. Por outro lado, dentro de um mesmo nível de $\mathrm{F}$ nota-se o aumento proporcional de $\mathrm{Hc}$ e $\mathrm{Mr}$, ao 
Influência da Energia de Soldagem do Processo RSW sobre as Propriedades Magnéticas e Tensões Residuais no Aço AISI 444

considerar um crescimento da energia de soldagem com exceção para o valor de Mr apresentado entre a amostra 2 e amostra 3. Este fato pode ser melhor justificado pelo o menor índice de correlação apresentado de $\mathrm{Mr}$ com relação à Hc para a energia de soldagem mostrados na Tabela 4. Conforme observado por Montgomery [17] é considerado como forte os valores de Correlação de Pearson $\geq 0,7$ e significativas (intervalo de confiança $\geq 95 \%$ ) os valores de P-Value $\leq 0,05$. Ambos contemplados na Figura 2a pelo posicionamento das linhas referentes as amostras estudadas, quanto mais longe do centro dos quadrantes estiverem a magnetização remanente Mr e o campo magnético coercivo Hc, maior são os valores dos sinais magnéticos apresentados. Neste sentido, limitações em termo de energia de soldagem devem ser levadas em consideração, principalmente para aplicações específicas como exemplo na soldagem de equipamentos biomédicos onde a elevação do campo magnético Hc acima de $10 \mathrm{G}$ pode inibir a função de equipamentos que mantém a vida humana [10]. Ao analisar as elevações dos níveis de Q na Tabela 3, observa-se na Figura 2b uma aparente queda dos valores de magnetização de saturação Ms em relação a amostra 0, mesmo a Tabela 4 não apresentando uma correlação forte e significativa entre ambas propriedades magnéticas.

Ainda na Tabela 4 os valores apresentados para a energia de soldagem $Q$ em relação à tensão residual $\sigma$ compressiva [18] não apresentam correlação forte e significativa, mantendo-se abaixo do limite determinado por

Tabela 3. Parâmetros magnéticos e de tensão residual obtidos após soldagem RSW.

\begin{tabular}{cccccccccccc}
\hline Amostra & $\mathbf{t}$ & $\mathbf{I}$ & $\mathbf{F}$ & $\mathbf{L}$ & $\mathbf{H} \mathbf{M r}$ & $\mathbf{M s}$ & $\boldsymbol{\mu}$ & $\mathbf{A}$ & $\boldsymbol{\sigma}$ & $\mathbf{Q}$ \\
& $\mathbf{S}$ & $\mathbf{A}$ & $\mathbf{N}$ & $\mathbf{m m}$ & $\mathbf{k G}$ & $\mathbf{e m u / g}$ & $\mathbf{e m u / g}$ & Adimensional & $\mathbf{k G . e m u / g}$ & $\mathbf{M P a}$ & Joule \\
\hline & - & - & - & - & 0,01345 & 1,045 & 169,38 & 12658739,15 & $-14345,02$ & $-231,3$ & - \\
1 & 0,166 & 4200 & 2000 & 0,13 & 0,01435 & 1,017 & 165,80 & 11626844,34 & $-15640,24$ & $-149,1$ & 215,25 \\
2 & 0,500 & 4200 & 2000 & 0,87 & 0,01542 & 1,244 & 167,00 & 10898815,29 & $-14960,63$ & $-54,9$ & 645,75 \\
3 & 0,166 & 5400 & 2000 & 1,02 & 0,01525 & 1,354 & 167,00 & 11022147,81 & $-16832,87$ & $-242,0$ & 355,82 \\
4 & 0,166 & 4200 & 3015 & 0,37 & 0,01508 & 1,279 & 165,47 & 11045953,92 & $-15684,53$ & $-277,3$ & 209,75 \\
5 & 0,500 & 4200 & 3015 & 1,34 & 0,01710 & 1,483 & 165,77 & 9765741,38 & $-18402,60$ & $-74,6$ & 629,27 \\
6 & 0,166 & 5400 & 3015 & 1,27 & 0,01623 & 1,391 & 165,90 & 10296622,94 & $-18565,61$ & $-42,0$ & 346,74 \\
7 & 0,500 & 5400 & 3015 & 1,49 & 0,01750 & 1,637 & 161,59 & 9305326,30 & $-19162,25$ & $-25,6$ & 1040,2 \\
\hline
\end{tabular}

Tabela 4. Correlações entre parâmetros de soldagem e propriedades magnéticas e de tensão.

\begin{tabular}{|c|c|c|c|c|c|c|c|c|c|c|}
\hline & $\mathbf{t}$ & I & $\mathbf{P}$ & Hc & $\mathrm{Mr}$ & Ms & $\mathbf{L}$ & $\boldsymbol{\mu}$ & A & $\boldsymbol{\sigma}$ \\
\hline I & $\begin{array}{c}-0,167 \\
0,721\end{array}$ & & & & & & & & & \\
\hline$P$ & $\begin{array}{l}0,167 \\
0,721\end{array}$ & $\begin{array}{l}0,167 \\
0,721\end{array}$ & & & & & & & & \\
\hline $\mathrm{Hc}$ & $\begin{array}{l}0,679 \\
0,094\end{array}$ & $\begin{array}{l}0,392 \\
0,384\end{array}$ & $\begin{array}{l}0,687 \\
0,088\end{array}$ & & & & & & & \\
\hline $\mathrm{Mr}$ & $\begin{array}{l}0,533 \\
0,218\end{array}$ & $\begin{array}{l}0,561 \\
0,191\end{array}$ & $\begin{array}{l}0,655 \\
0,103\end{array}$ & $\begin{array}{l}0,937 \\
0,002\end{array}$ & & & & & & \\
\hline Ms & $\begin{array}{c}-0,368 \\
0,417\end{array}$ & $\begin{array}{c}-0,343 \\
0,451\end{array}$ & $\begin{array}{c}-0,560 \\
0,191\end{array}$ & $\begin{array}{c}-0,633 \\
0,127\end{array}$ & $\begin{array}{c}-0,616 \\
0,141\end{array}$ & & & & & \\
\hline L & $\begin{array}{l}0,563 \\
0,188\end{array}$ & $\begin{array}{l}0,609 \\
0,147\end{array}$ & $\begin{array}{l}0,464 \\
0,295\end{array}$ & $\begin{array}{l}0,906 \\
0,005\end{array}$ & $\begin{array}{l}0,906 \\
0,005\end{array}$ & $\begin{array}{c}-0,376 \\
0,405\end{array}$ & & & & \\
\hline$\mu$ & $\begin{array}{c}-0,662 \\
0,105\end{array}$ & $\begin{array}{l}-0,411 \\
0,359\end{array}$ & $\begin{array}{c}-0,709 \\
0,075\end{array}$ & $\begin{array}{r}-0,977 \\
0,000\end{array}$ & $\begin{array}{c}-0,944 \\
0,001\end{array}$ & $\begin{array}{l}0,682 \\
0,092\end{array}$ & $\begin{array}{r}-0,891 \\
0,007\end{array}$ & & & \\
\hline$A$ & $\begin{array}{c}-0,264 \\
0,567\end{array}$ & $\begin{array}{c}-0,643 \\
0,120\end{array}$ & $\begin{array}{c}-0,683 \\
0,091\end{array}$ & $\begin{array}{c}-0,866 \\
0,012\end{array}$ & $\begin{array}{c}-0,833 \\
0,020\end{array}$ & $\begin{array}{l}0,604 \\
0,151\end{array}$ & $\begin{array}{c}-0,826 \\
0,022\end{array}$ & $\begin{array}{l}0,867 \\
0,011\end{array}$ & & \\
\hline$\sigma$ & $\begin{array}{l}0,664 \\
0,104\end{array}$ & $\begin{array}{l}0,189 \\
0,685\end{array}$ & $\begin{array}{l}0,231 \\
0,618\end{array}$ & $\begin{array}{l}0,651 \\
0,113\end{array}$ & $\begin{array}{l}0,419 \\
0,350\end{array}$ & $\begin{array}{c}-0,373 \\
0,410\end{array}$ & $\begin{array}{l}0,627 \\
0,132\end{array}$ & $\begin{array}{c}-0,639 \\
0,122\end{array}$ & $\begin{array}{c}-0,515 \\
0,237\end{array}$ & \\
\hline $\mathrm{Q}$ & $\begin{array}{l}0,874 \\
0,010\end{array}$ & $\begin{array}{l}0,278 \\
0,546\end{array}$ & $\begin{array}{l}0,269 \\
0,559\end{array}$ & $\begin{array}{l}0,814 \\
0,026\end{array}$ & $\begin{array}{l}0,755 \\
0,050\end{array}$ & $\begin{array}{c}-0,673 \\
0,098\end{array}$ & $\begin{array}{l}0,733 \\
0,061\end{array}$ & $\begin{array}{l}-0,821 \\
0,024\end{array}$ & $\begin{array}{c}-0,533 \\
0,218\end{array}$ & $\begin{array}{l}0,690 \\
0,086\end{array}$ \\
\hline
\end{tabular}



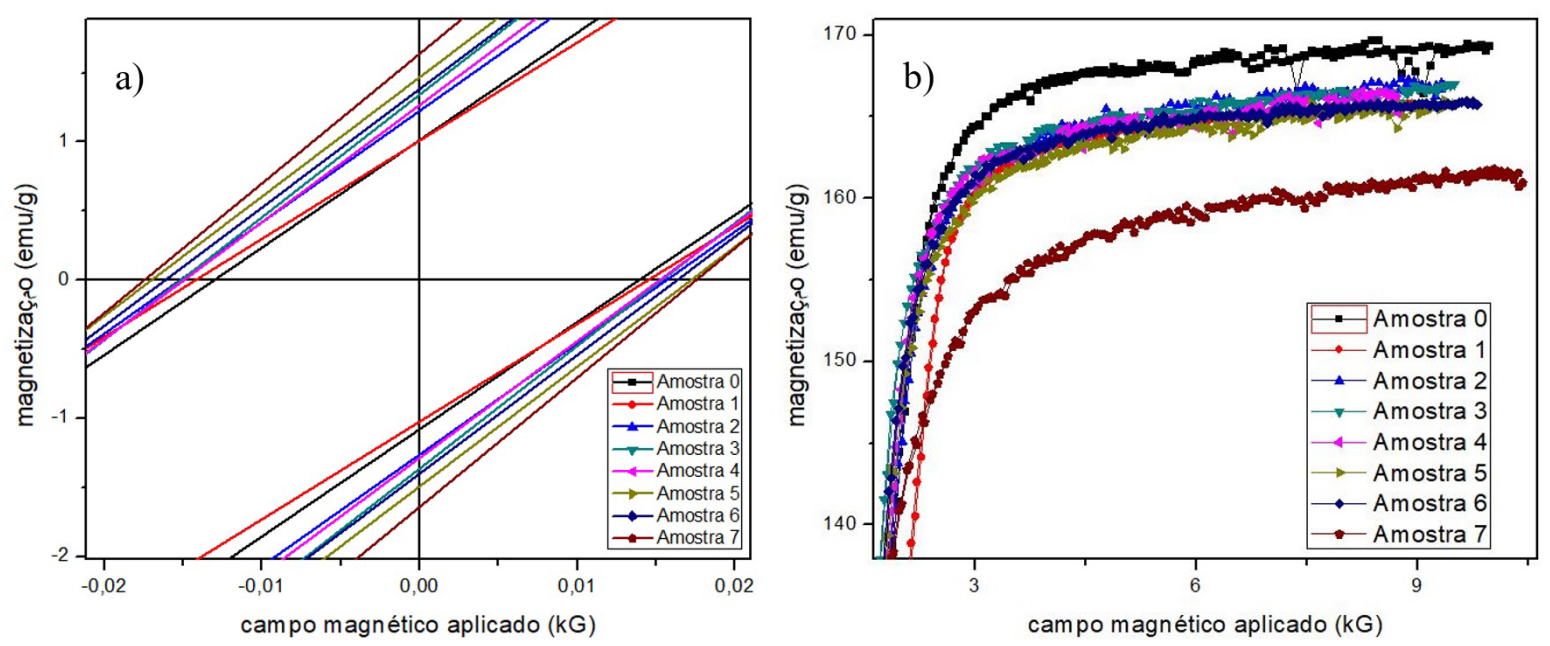

Figura 2. Histerese: a) campo magnético coercivo Hc e magnetização remanente Mr, b) magnetização de saturação Ms.

Montgomery [17]. Neste sentido, Vashista \& Paul [7] constataram que $\sigma$ é uma propriedade sensível a Ms e $\mu$, mesmo não apresentando correlação forte e significativa de $\sigma$ com Ms, A e $\mu$. Talvez uma amostragem maior aliada a novas técnicas mais sensíveis de medição magnética com elevadas frequências [7], represente melhor estas correlações descritas anteriormente. A permeabilidade magnética $\mu$ indica o quanto um material é permeável quando submetido a um campo magnético externo. Geralmente, uma alta permeabilidade induz a um alto magnetismo por meio de um pequeno campo magnético aplicado. Esta característica magnética, pode sofrer alterações durante a soldagem RSW por possuir uma forte e significante correlação inversamente proporcional (pelo seu valor negativo) com a deformação $L$ e com a energia de soldagem $Q$. A correlação entre a deformação $L$ e as propriedades magnéticas $\mathrm{Hc}$ e $\mathrm{Mr}$ além de proporcionais pelo seu valor positivo, são mais fortes e significativas em relação a Q. Já para os valores de correlação entre $\mathrm{L}$ com $\mathrm{Q}$ e de $\mathrm{P} \operatorname{com} \mu$, observa-se que ambos apresentam correlação forte mesmo tendo uma baixa significância caracterizada pelo seu P-Value $\geq 0,05$.

A microestrutura da seção transversal do ponto soldado foi melhor revelada pela ação do ácido oxálico, eleito o mais adequado para esta pesquisa. Desta forma, foi possível observar na Figura 3a uma ampliação dos precipitados de $\mathrm{Nb}$ e Ti que tem suas composições químicas claramente detalhadas nas Figuras $3 \mathrm{~b}$ e $3 \mathrm{~d}$. Estes precipitados apresentam-se aparentemente em maior quantidade na zona termicamente afetada (ZTA) do aço AISI 444 conforme mostrado na Figura 3c. Este fenômeno, pode ser devido ao aquecimento e resfriamento muito rápido presente no processo de soldagem RSW. Assim, nem todos os precipitados são completamente dissolvidos e podem se precipitar na ZTA antes da solidificação do ponto de solda. Neste caso, os tamanhos dos precipitados de Ti e Nb em forma de carbonetos finos além de impedir a sensitização, também melhoram a soldabilidade [8] ao limitar a formação de martensita, carbonitretos de cromo e o crescimento dos grãos. Por outro lado, muito embora os precipitados possam colaborar para o aumento da dureza mecânica [19] e magnética [8], podem tornar-se um fator complicador na utilização do aço AISI 444 em equipamentos onde a característica magnética mole faz-se necessário [11].

$\mathrm{Na}$ Figura 4, a macrografia do ponto soldado referente à amostra 1 revela detalhes interessantes. Constata-se que, próximo ao centro do ponto de soldagem os grãos apresentam-se maiores em relação a ZTA e ao metal base, conforme observado por Alizadeh [20].

É notável a formação de grãos colunares no centro da zona fundida representados nas Figuras 4a e 4b. Este fenômeno pode ser explicado pela maior taxa de resfriamento radial ante axial [21] aliado ao baixo valor de L. Em contrapartida, à medida que a deformação $L$ aumenta, os grãos colunares ficam cada vez menores no centro da zona fundida conforme apresentado nas Figuras 5a e 5b. Muito embora Moshayedi [18] tenha constatado que o aumento de L possa acarretar um aumento da $\sigma$ de compressão, a Tabela 4 não apresentou correlação forte $e$ significativa entre $\sigma$ e L. 
Influência da Energia de Soldagem do Processo RSW sobre as Propriedades Magnéticas e Tensões Residuais no Aço AISI 444
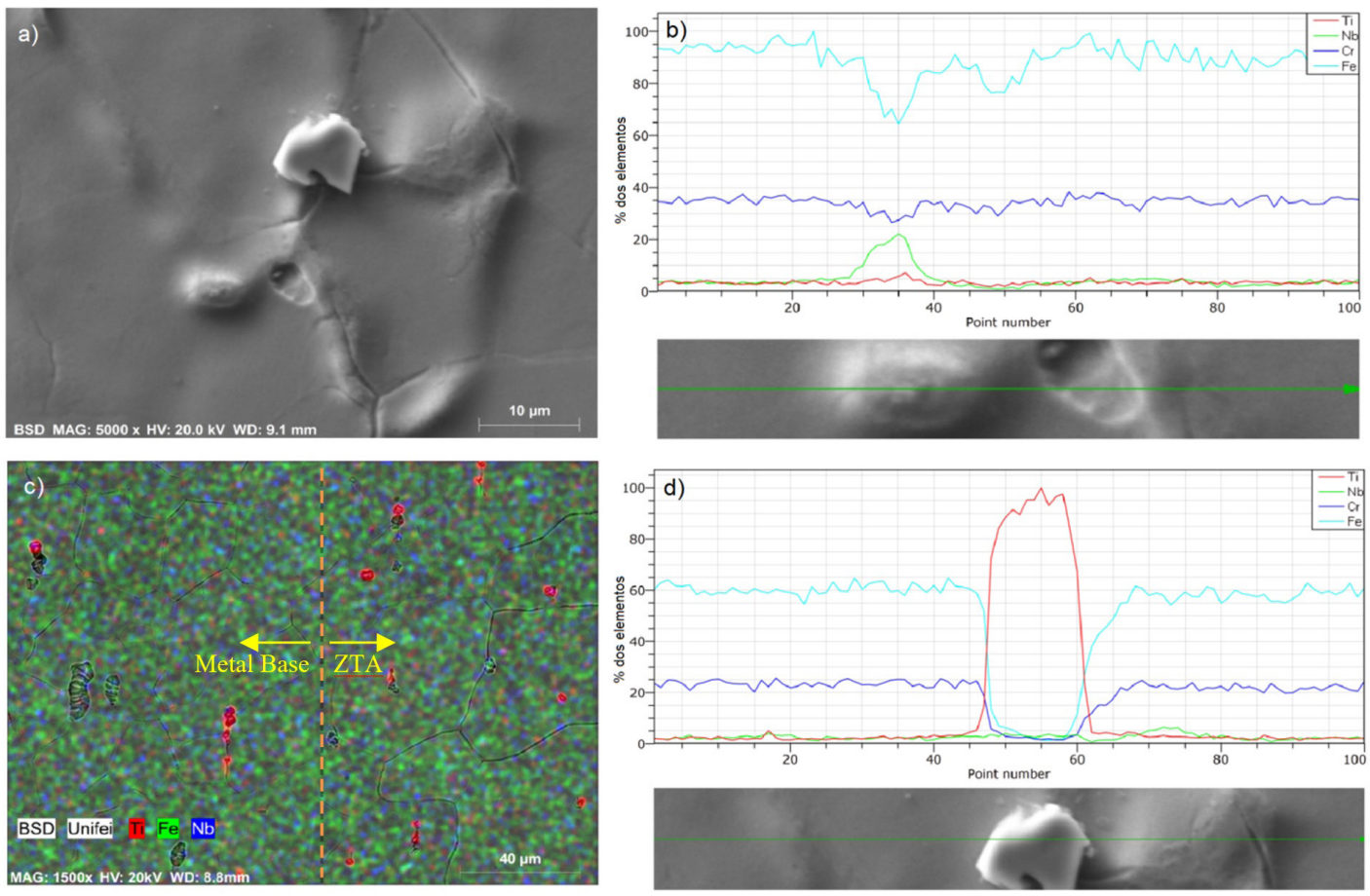

Figura 3. MEV da seção transversal do ponto de solda da amostra 3: a) ampliação 5000x de um precipitado, b) e d) análise química dos precipitados, c) macrografia dos precipidados por setor.
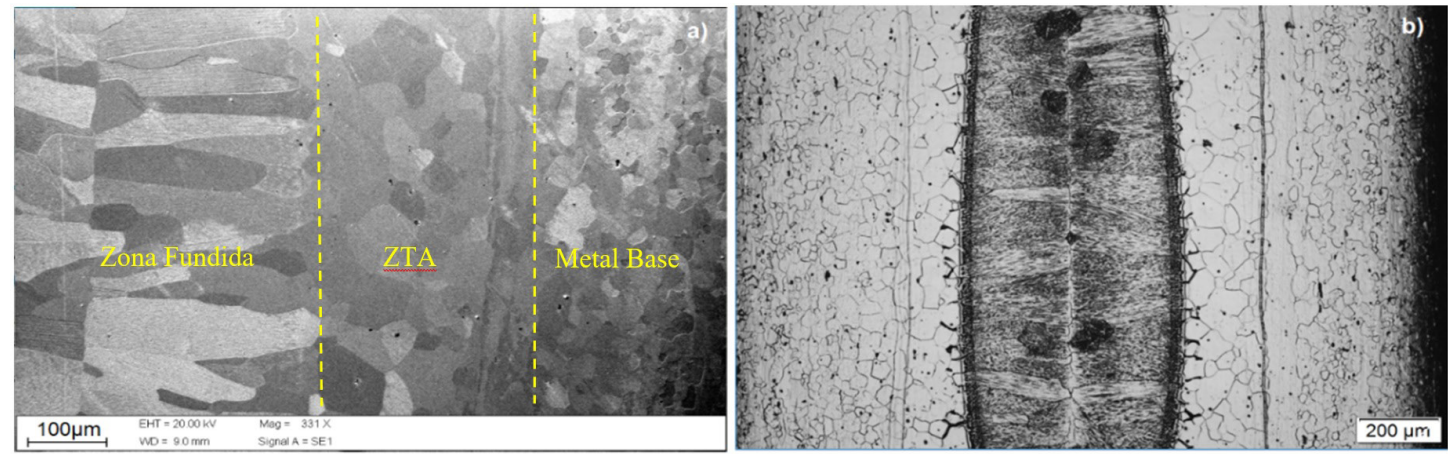

Figura 4. a) Ampliação 300x do centro da zona fundida da amostra 1, b) macrografia da seção transversal do ponto de solda da amostra 1.
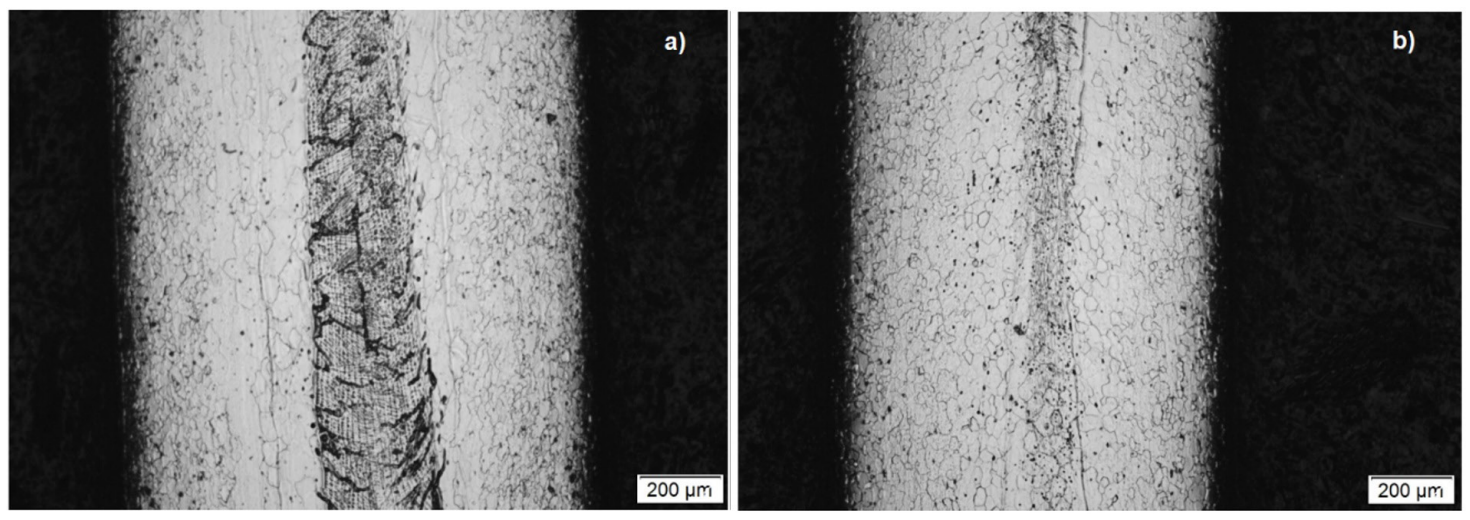

Figura 5. Macrografia da seção transversal do ponto de solda: a) amostra 2, b) amostra 3. 
Observando as Figuras $5 \mathrm{~b}$ e a $6 a$ percebe-se que a zona de fusão no centro da imagem é mais estreita em relação à apresentada na Figura 5 a. Mesmo $P$ apresentando correlação forte e não significativa $\operatorname{com} \mu$, a variação de L apresentada nas amostras 3 (Figura 5b) e 6 (Figura 6a) pode ter sido suficiente para provocar o estreitamento da zona de fusão e variar as propriedades magnéticas. Já para amostra 7 ocorre uma deformação maior da zona fundida, quando os grãos crescidos (Figura $4 \mathrm{~b}$ e $5 \mathrm{a}$ ) dão lugar a grãos pequenos e aparentemente alongados mostrados na Figura 6b. Neste caso, o baixo valor de Ms apresentado pode ser devido ao menor deslocamento da parede de domínios magnéticos na direção do campo magnético aplicado [22].
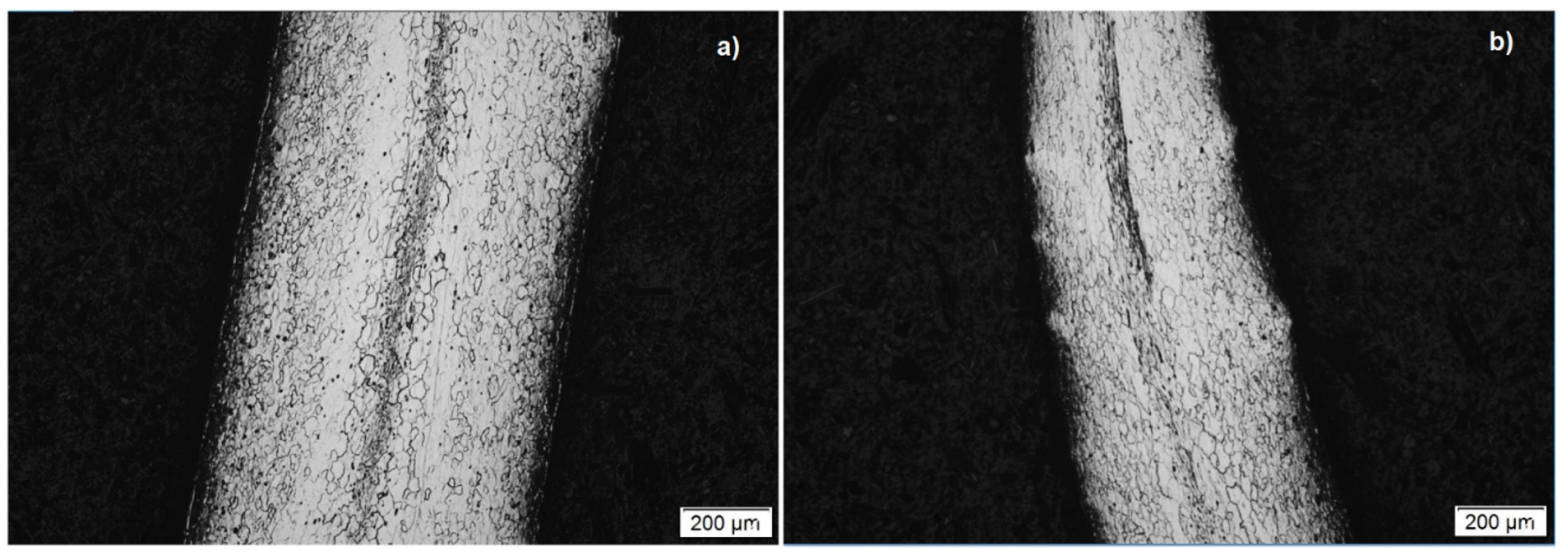

Figura 6. Macrografia da seção transversal do ponto de solda: a) amostra 6, b) amostra 7.

\section{Conclusão}

As análises mostraram que a energia de soldagem afeta as propriedades magnéticas $\mathrm{Hc}$, $\mathrm{Mr}$ e $\mu$, mas não possui correlação forte e significativa com a tensão residual. Por outro lado, há uma maior influência da deformação frente à energia de soldagem sobre as propriedades magnéticas de $\mathrm{Hc}, \mathrm{Mr}, \mu$ e A. Este contexto reforça a possibilidade da utilização do sensor de deslocamento do eletrodo como bom indicador da variação das propriedades magnéticas dentro da soldagem RSW. Para baixos valores de deformação os grãos da zona fundida tendem a alongar-se. 0 baixo nível de Ms apresentado na amostra 7 em relação as demais, pode ser justificado pela grande deformação nos grãos do ponto soldado. A permeabilidade magnética pode ser considerada um importante indicador magnético devido à forte correlação com $\mathrm{Q}, \mathrm{L}$ e $\mathrm{P}$ mesmo sendo para este último não significante. Entende-se para tanto que, as correlações apresentadas neste trabalho abrem caminho para uma avaliação sobre a possibilidade de otimização dos parâmetros de soldagem com intuito de minimizar a variação magnética do metal pós-soldagem. A correlação entre $Q, \sigma$ e as propriedades magnéticas do material soldado talvez possam apresentar resultados mais fortes $\mathrm{e}$ significativos ao utilizar frequências maiores na medição magnética e de tensão residual.

\section{Agradecimentos}

À UNIFEl pela infraestrutura laboratorial. Da mesma forma a CNPQ, a APERAM, PRATICA FORNOS, CAPES e a FAPEMIG.

\section{Referências}

[1] Baldim AC, Costa SC, Aguiar TCS. Metodologia não destrutiva de parametrização da queima do zinco no processo de soldagem a ponto por resistência. Soldagem e Inspeção. 2015;20(1):81-91. http://dx.doi.org/10.1590/0104-9224/SI2001.09.

[2] Nascimento VC. Seleção de parâmetros de soldagem a ponto por resistência, baseado na medição da resistência elétrica entre chapas [dissertação de mestrado]. Uberlândia: Faculdade de Engenharia Mecânica, Universidade Federal de Uberlândia; 2008.

[3] Folkhard E. Welding metallurgy of stainless steels. New York: Spring-Verlag Wien; 1988. http://dx.doi.org/10.1007/978-37091-8965-8.

[4] Lippold JC, Kotecki DJ. Welding metallurgy and weldability of stainless steels. Hoboken: John Willey \& Sons; 2005. 
Influência da Energia de Soldagem do Processo RSW sobre as Propriedades Magnéticas e Tensões Residuais no Aço AISI 444

[5] Baddoo NR. Stainless steel in construction: a review of research, applications, challenges and opportunities. Journal of Constructional Steel Research. 2008;64(11):1198-1206. http:// dx.doi.org/10.1016/j.jcsr.2008.07.011.

[6] Australian Stainless Steel Development Association. Magnetic effects of stainless steel. Brisbane: ASSDA; 2007. (Technical FAQs; 3).

[7] Vashista M, Paul S. Correlation between surface integrity of ground medium carbon steel with Barkhausen Noise parameters and magnetic hysteresis loop characteristics. Materials \& Design. 2009;30(5):1595-1603. http://dx.doi.org/10.1016/j. matdes.2008.07.030.

[8] Tavares SSM, Souza JA, Herculano LFG, Abreu HFG, Souza CM Jr. Microstructural, magnetic and mechanical property changes in an AISI 444 stainless steel aged in the $560^{\circ} \mathrm{C}$ to $800^{\circ} \mathrm{C}$ range. Materials Characterization. 2008;59(2):112-116. http://dx.doi. org/10.1016/j.matchar.2006.11.002.

[9] Malheiro VN, Spear RL, Brooks RA, Markaki AE. Osteoblast and monocyte responses to 444 ferritic stainless steel intended for a magneto-mechanically actuated fibrous scaffold. Biomaterials. 2011;32(29):6883-6892. http://dx.doi.org/10.1016/j. biomaterials.2011.06.002. PMid:21703680.

[10] Martín Ó, López M, De Tiedra P, Juan MS. Prediction of magnetic interference from resistance spot welding processes on implantable cardioverter-defibrillators. Journal of Materials Processing Technology. 2008;206(1-3):256-262. http://dx.doi. org/10.1016/j.jmatprotec.2007.12.021.

[11] Meyer N, Mantel M, Dubois PE, Veron M, Brechet Y, Geoffroy O. Influence of $\mathrm{Nb}$ stabilization on the recovery and recrystallization kinetics of a ferritic stainless steel with soft magnetic properties for automotive applications. R. Esc. Minas, Ouro Preto. 2013;66(2):159-163.

[12] CEGLIAS, R. B. Análise de tensão residual em tubo de aço API $5 \mathrm{~L}$ X70 [dissertação de mestrado]. Rio de Janeiro: Faculdade de Engenharia, Instituto Militar de Engenharia; 2012.

[13] Modenesi PJ. Soldabilidade dos aços inoxidáveis. Vol. 1. São Paulo: SENAI-SP; 2001.
[14] Silva EM. Estudo da correlação entre o aporte térmico, as propriedades magnéticas e a corrosão sob tensão em juntas soldadas de aço inoxidável ferrítico AISI-409 [tese de doutorado]. Itajubá: Instituto de Engenharia Mecânica, Universidade Federal de Itajubá; 2011.

[15] Tan W, Zhou Y, Kerr HW, Lawson S. A study of dynamic resistance during small scale resistance spot welding of thin Ni sheets. Journal of Physics. D, Applied Physics. 2004;37(14):1998-2008. http://dx.doi.org/10.1088/0022-3727/37/14/017.

[16] Material Property Data [página da internet]. Blacksburg: MatWeb; 2017 [acesso em 09 mar. 2017]. Disponível em: http://www. matweb.com

[17] Montgomery DC. Design and analysis of experiments. New York: John Wiley, 2005.

[18] Moshayedi H, Sattari-Far I. Resistance spot welding and the effects of welding time and current on residual stresses. Journal of Materials Processing Technology. 2014;214(11):2545-2552. http://dx.doi.org/10.1016/j.jmatprotec.2014.05.008.

[19] Kwun H, Burkhardt GL. Effects of grain size, hardness, and stress on the magnetic hysteresis loops of ferromagnetic Steels. Journal of Applied Physics. 1987;61(4):1-4. http://dx.doi. org/10.1063/1.338093.

[20] Alizadeh-Sh M, Pouranvari M, Marashi SPH. Welding metallurgy of stainless steels during resistance spot welding part II: heat affected zone and mechanical performance. Science and Technology of Welding and Joining. 2015;20(6):512-521. http:// dx.doi.org/10.1179/1362171815Y.0000000010.

[21] Wei PS, Wu TH. Magnetic property effect on transport processes in resistance spot welding. Journal of Physics. D, Applied Physics. 2011;44(32):10. http://dx.doi.org/10.1088/00223727/44/32/325501.

[22] Ribeiro VAS, Rodrigues G, Pereira AC, Oliveira AF, Mendonça CSP, Oliveira VD, et al. Investigação das propriedades magnéticas e microestrutura da ferrita de chumbo e cobre. Cerâmica. 2016;62(361):98-104. http://dx.doi.org/10.1590/036669132016623611927. 